\title{
Buckling of polysilicon microbeams during sacrificial layer removal
}

\author{
Tong-Yi Zhang $\dagger$, Xin Zhang and Yitshak Zohar \\ Department of Mechanical Engineering, Hong Kong University of Science and \\ Technology, Clear Water Bay, Kowloon, Hong Kong
}

Received 16 March 1998, accepted for publication 6 May 1998

\begin{abstract}
In situ observations of buckling evolution of polysilicon microbeams during etch of the underneath sacrificial layer were carried out under an optical microscope. The surface geometry was obtained by AFM measurements. As the etching progressed, three buckling patterns were identified. Closed formulas were derived from theoretical analysis based on both boundary conditions: simply supported and clamped. The theory predicts either the buckling pattern for a given residual stress or the compressive stress level for a given buckling pattern. The residual stress evaluated from the buckling pattern agrees with that measured by the curvature method.
\end{abstract}

\section{Introduction}

Microelectromechanical systems (MEMS) have achieved impressive progress and become a very active area of research as they could potentially lead to another industrial revolution [1]. Thin film materials used in the fabrication of MEMS may have different properties from their bulk counterparts. Residual stresses, usually introduced during thin film deposition, can damage micromechanical devices during fabrication or reduce their service life. Moreover, many of the most important failure mechanisms in integrated circuits such as stress voiding or delamination are mechanical in nature. Compressive residual stress above a critical level results in buckling, but the development of the buckling phenomenon in micromachines requires studies on micromechanical structures. Hutchinson et al [2] and Jensen and Thouless [3] have investigated buckling-driven film delamination theoretically and experimentally. They designed a model system consisting of a mica film bonded to an aluminum substrate. Owing to the thermal-expansion mismatch between mica and aluminum, an equi-biaxial, compressive stress was induced in the bonded portion of the mica due to the cooling from the bonding temperature. When the initial delamination is sufficiently large, the film buckles away from the substrate producing a blister which in turn induces a driving force on the interface crack tip. Their results indicate that if crack growth occurs, buckling will cause a wavy crack front to develop. They measured a single post-buckling profile under the influence of contact between the film and substrate. However, this profile is highly irregular in both phase and amplitude. Furthermore, no measurements of the surface geometry are available.

A theoretical analysis of problems such as buckling of thin films or blister test of a thin film always involves

$\dagger$ Corresponding author. Tel.: (852) 2358-7192. Fax: (852) 2358-1543. E-mail: mezhangt@usthk.ust.hk. the difficulty of which boundary condition to use for the bonded edges of the film. In general, two kinds of boundary condition have widely been used in the literature: either simply supported or clamped boundary conditions. Vlassak and Nix [4] adopted simply supported boundary conditions in their work on the blister testing. On the other hand, Jensen and Thouless [3] have used clamped-edge boundary conditions in their classical buckling and post-buckling analysis of the mica film-aluminum substrate system.

In surface micromachining, the thickness of films formed on the silicon substrate by a variety of techniques is on the order of 1 micron. When the film is debonded or freed from the sacrificial layer, it is not that clear which boundary condition better describes the physical reality at the attached film edge. In this work, using micromachining techniques, we fabricated microbeams and carried out in situ observations of the buckling patterns. Furthermore, we calculated the buckling profile based on the simply supported as well as the clamped boundary conditions. Consequently, the comparison between the experimental measurements and the theoretical predictions is much improved.

\section{Experiment}

A p-type (100) silicon wafer was used as a substrate in the present study. The process started with the deposition of a $0.42 \mu \mathrm{m}$ low-temperature oxide (LTO) layer in a LPCVD reactor with $\mathrm{SiH}_{4}$ and $\mathrm{O}_{2}$ as the source gases at $425^{\circ} \mathrm{C}$. The wafer was then annealed in a standard diffusion furnace in $\mathrm{N}_{2}$ atmosphere at $900^{\circ} \mathrm{C}$ for 1 hour. The oxide on the wafer was patterned and etched down to the silicon wafer to form the anchor areas. Next, a uniform layer of polycrystalline silicon, $0.6 \mu \mathrm{m}$ thick, was deposited in another LPCVD reactor at $620^{\circ} \mathrm{C}$. After stripping the 


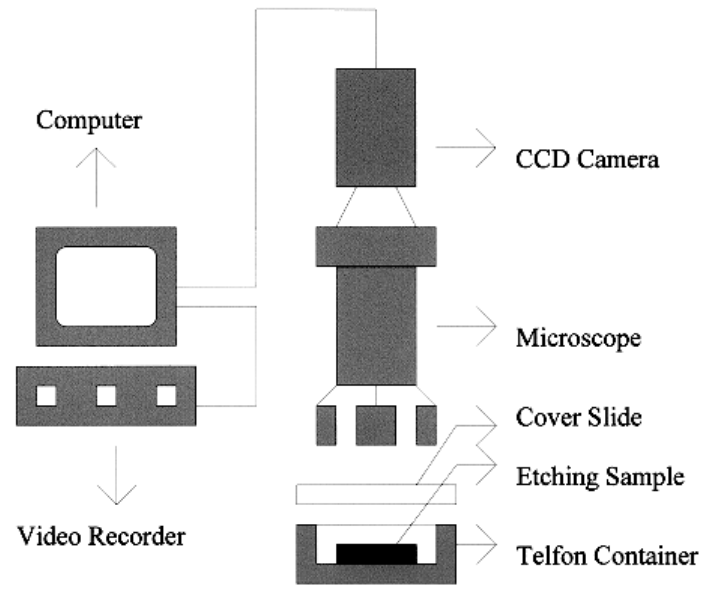

Figure 1. Experimental set-up for in situ observation of buckling.

polysilicon layer on the back side, the residual stress on the front-side polysilicon layer was measured using an SMSi 3800 wafer curvature system and it was found to be compressive and about $315 \mathrm{MPa}$. Finally, the polysilicon layer was patterned and etched in an $\mathrm{SF}_{6}: \mathrm{CClF}_{5}$ plasma reactor to form the doubly anchored beams. The wet etch of the LTO sacrificial layer was conducted in an HF solution at room temperature and monitored in situ by the specially designed video system, as shown in figure 1 .

\section{Results and discussion}

The mid-plane of the microbeam is horizontal and contains the $x$ and $y$ axes, while the $z$ axis is directed vertically upward, as shown in figure 2. The two parallel straight lines along the beam, denoted as FLE, are the etch fronts indicating the extent of the etched sacrificial layer. $L_{e}$ denotes the etching length, which increases with etching time. The dark area between the two etch fronts is still bonded to the substrate via the sacrificial layer. Figures 3(a) and 3(b) show the evolution of the buckling pattern of the microbeams in the etching solution. In general, there are three different modes of buckling pattern during the etching process. The first mode of buckling pattern, figure 3(a), occurred in the early stage of etching, where the Newton rings for the convex part have the same size as those for the concave part. Figure 3(b) shows the second mode of buckling pattern, that developed from the first mode as the etching proceeded. In this second mode, the size of the Newton rings for the convex part is larger than that for the concave part. The third mode of buckling is the classical one [5], which appeared when the sacrificial layer was completely etched away while the microbeam was still immersed in the etching solution. After etching and during the drying process, a new mode of buckling, i.e., blister-like local buckling, developed and its details have been reported elsewhere [6]. Three-dimensional surface profiles of the first and second modes of buckling patterns were measured using an atomic force microscope (AFM) on samples in which the etching process was stopped to freeze the temporal buckling pattern.

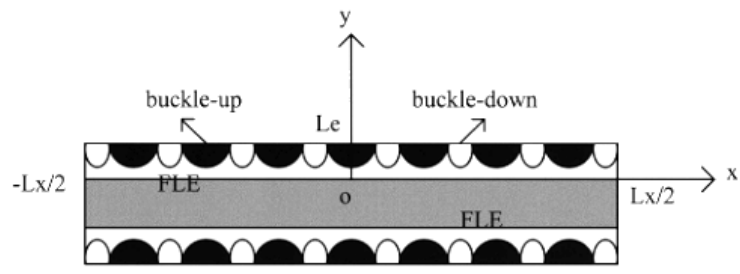

Figure 2. Schematic illustration of the buckling pattern of a partially etched microbeam and the associated coordinate system.

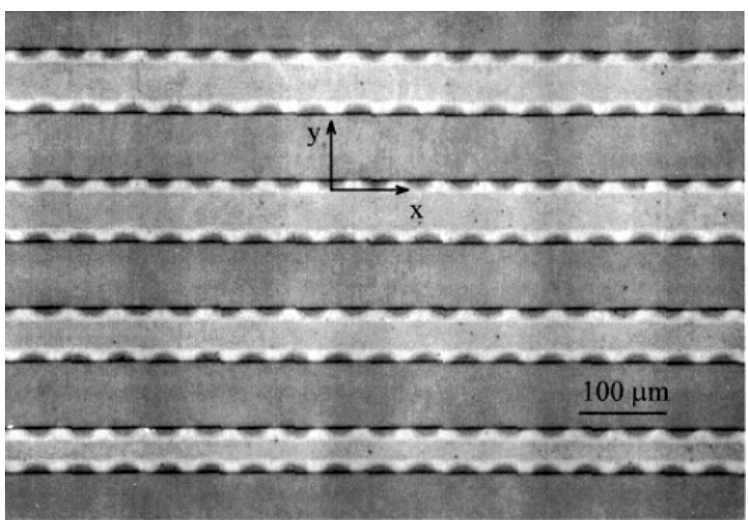

(a)

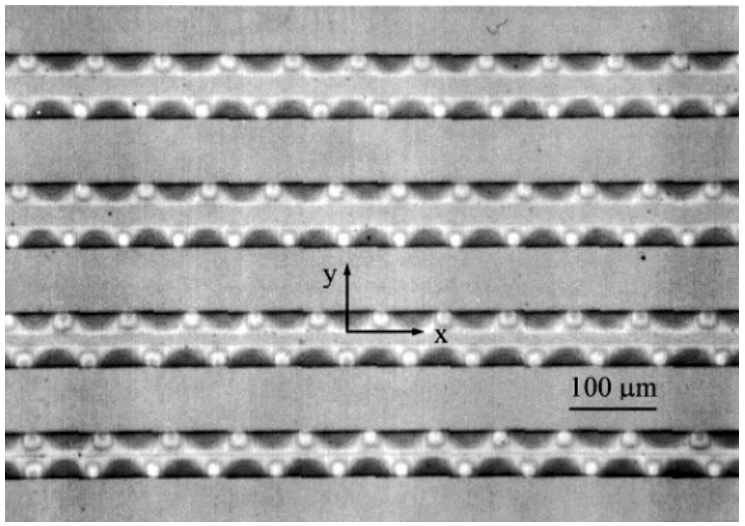

(b)

Figure 3. (a) Plan view of the first mode buckling. (b) Plan view of the second mode buckling.

Figures 4 and 5 show the AFM profile for both the first and second modes of buckling. The two-dimensional buckling patterns measured by AFM are given in 4(a) and 5(a), and the corresponding profiles are drawn in 4(b) and 5(b). A, B and C denote three typical profiles along horizontal or vertical directions. For the first mode of buckling, the profile along the free edge of the beam can be fitted by a sinusoidal function $A \sin (\lambda x)$, where $A$ is the amplitude and $2 \pi / \lambda$ is the wavelength. Therefore, the first mode of buckling is named the sinusoidal halfwaves. For the buckling pattern shown in figure 4, the amplitude and wavelength of the sine profile are $0.3 \mu \mathrm{m}$ and $50 \mu \mathrm{m}$, respectively. The amplitude of the sinusoidal profile is a function of the etching length $L_{e}$, and it increased with etching time until it reached the thickness of the sacrificial layer. With continuous etching, the buckling 


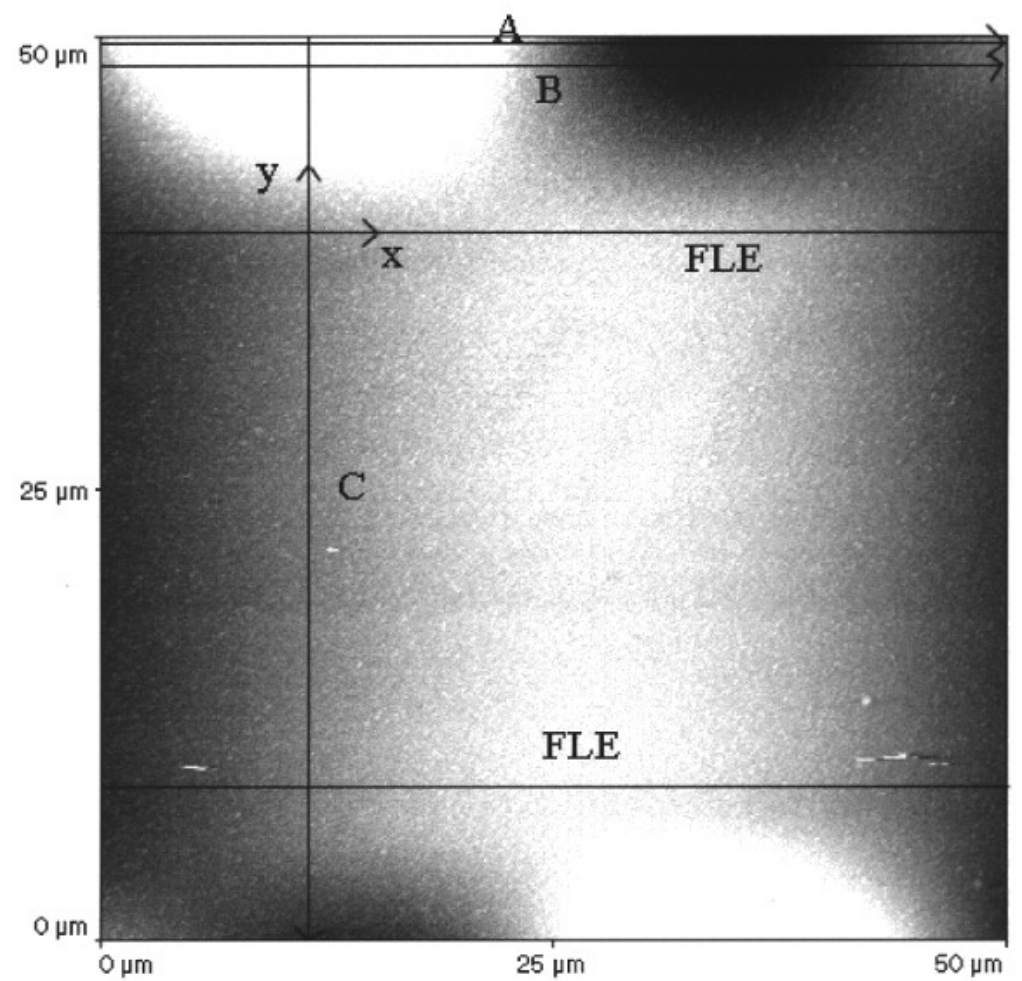

(a)

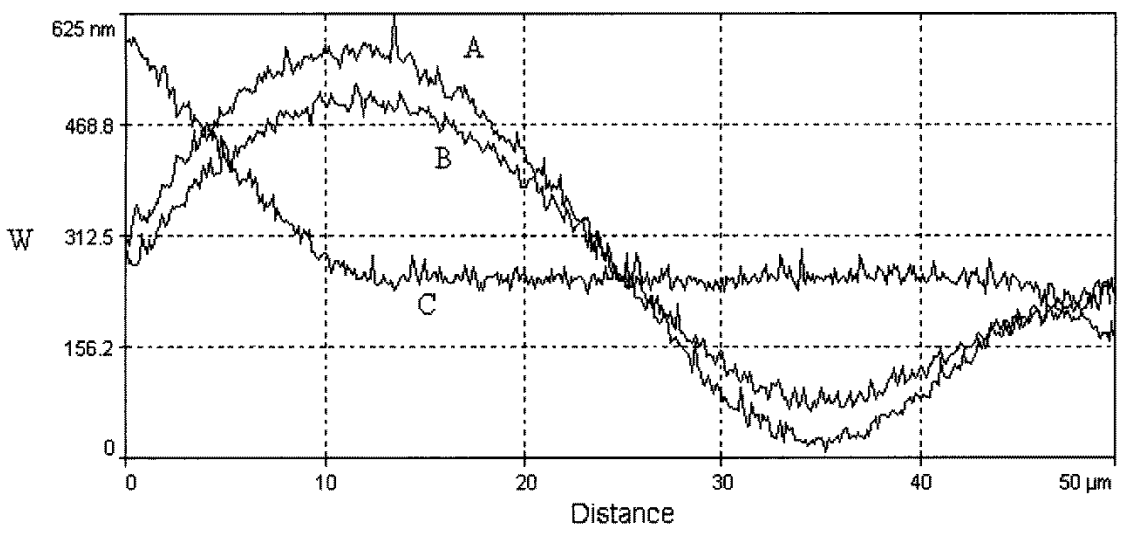

(b)

Figure 4. AFM profile of the first mode buckling, (a) tracing path and (b) profile.

pattern evolved into the second mode shown in figure 5 . In this mode, the height of the concave part was limited to the $0.42 \mu \mathrm{m}$ thickness of the sacrificial layer, while the height of the convex part was free to increase with etching time. Tracing path B in figure 5(b) indicates that the contact zone is not flat and maintains the sinuous shape. Thus, we call the second mode of buckling pattern constrainedsinusoidal waves. The buckling profile along the beam free edge was approximately fitted by a sinusoidal function of $\left[A \sin (\lambda x)+\left(A-h_{s}\right)\right]$, where $h_{s}$ is the thickness of the sacrificial layer. For the buckling pattern shown in figure 5, the amplitude and wavelength of the sinusoidal profile are, respectively, $0.55 \mu \mathrm{m}$ and $60 \mu \mathrm{m}$.

For the case of buckling under residual stresses, we assume that there is no bending load and no shear stress. The etching starts from the microbeam edges and hence the residual stress along the $y$ axis in the free part of the microbeam was totally or partially released, and is assumed to be zero in the present work for simplicity. It is reasonable to adopt the following boundary conditions: (1) simply-supported-edge boundary conditions at the two anchors of the microbeam, $x= \pm L_{x} / 2$, and (2) freeedge boundary conditions along the free edge of the microbeam, $y=L_{e}$ [5]. Along the etch front $y=0$, however, either simply-supported-edge or clamped-edge boundary conditions can be used. First, consider the simply-supported-edge boundary conditions. In general, there are three possible solutions that satisfy the governing 


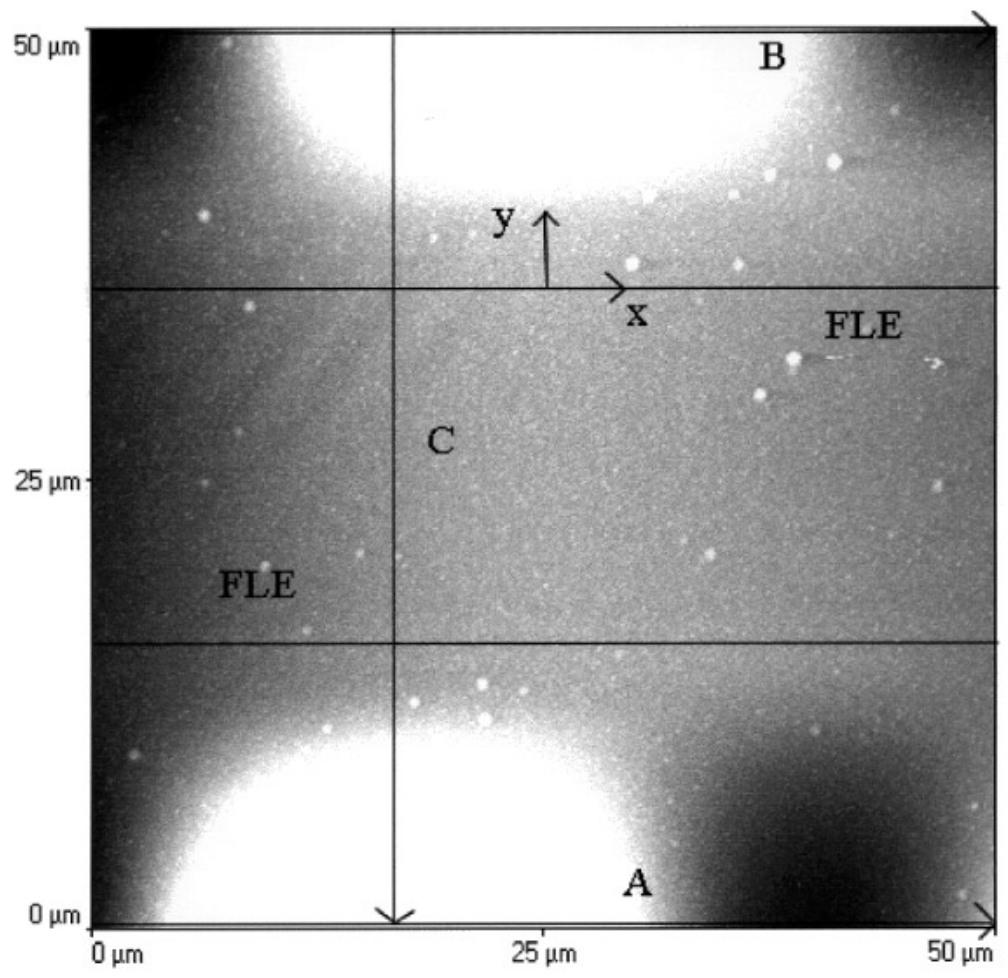

(a)

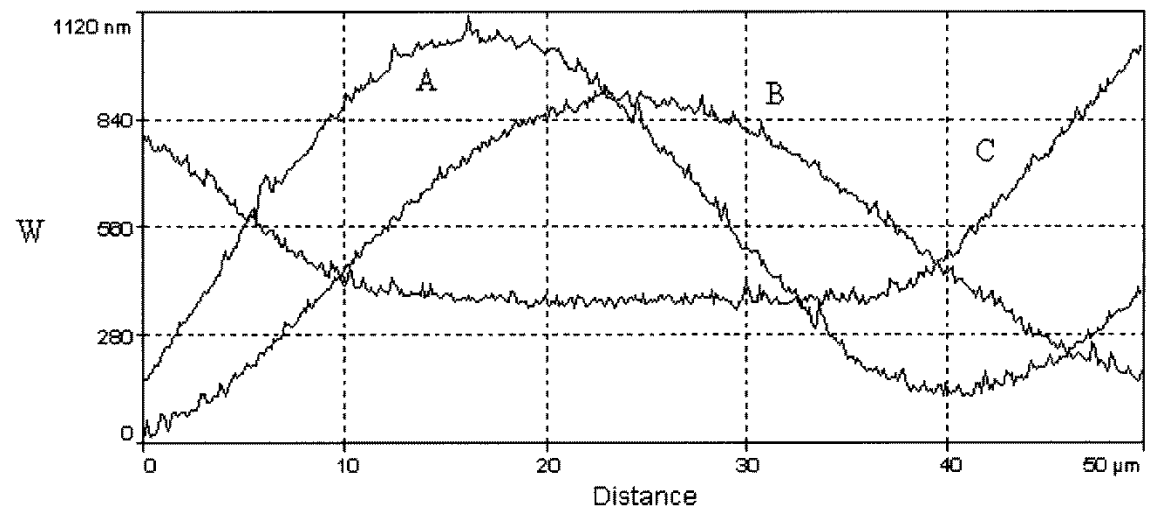

(b)

Figure 5. AFM profile of the second mode buckling, (a) tracing path and (b) profile.

equation and the boundary conditions. The three solutions are controlled by the relative magnitude of two parameters, $\lambda_{m}$ and $\sqrt{-\sigma_{x x} h / D}$, where $\sigma_{x x}$ is the residual stress along the $x$ axis, and $D$ is the flexural rigidity of the microbeam. $\lambda_{m}$ and $D$ are defined as

$$
\begin{aligned}
D & =\frac{E h^{3}}{12\left(1-v^{2}\right)} \\
\lambda_{m} & =\frac{m \pi}{L_{x}}
\end{aligned}
$$

where $m$ is the buckling circle number, $h$ is the thickness of the microbeam, $E$ and $v$ are respectively Young's modulus and the Poisson ratio of the beam. For $\lambda_{m}<\sqrt{-\sigma_{x x} h / D}$, the most common case for microbeam buckling, the displacement along the $z$ axis, $w$, is given by:

$$
\begin{aligned}
w & =A_{I}\left[\sinh \left(\alpha_{1} y\right)\right. \\
& \left.+\frac{\left(\alpha_{1}^{2}-v \lambda_{m}^{2}\right) \sinh \left(\alpha_{1} L_{e}\right)}{\left(\alpha_{2}^{2}+v \lambda_{m}^{2}\right) \sin \left(\alpha_{2} L_{e}\right)} \sin \left(\alpha_{2} y\right)\right] \sin \left(\lambda_{m} x\right)
\end{aligned}
$$

where $A_{I}$ is a constant having a relationship with the amplitude of buckling, $A$,

$$
A=A_{I} \sinh \left(\alpha_{1} L_{e}\right)\left(1+\frac{\alpha_{1}^{2}-v \lambda_{m}^{2}}{\alpha_{2}^{2}+v \lambda_{m}^{2}}\right)
$$


and

$$
\begin{aligned}
& \alpha_{1}^{2}=\lambda_{m}^{2}+\lambda_{m} \sqrt{-\sigma_{x x} h / D} \\
& \alpha_{2}^{2}=\lambda_{m}^{2}-\lambda_{m} \sqrt{-\sigma_{x x} h / D} .
\end{aligned}
$$

The residual stress $\sigma_{x x}$ has a negative sign and (3) is subject to the condition of

$$
\begin{aligned}
& \left(\alpha_{1}^{2}-v \lambda_{m}^{2}\right)\left[\alpha_{2}^{2}+(2-v) \lambda_{m}^{2}\right] \alpha_{2} \tanh \left(\alpha_{1} L_{e}\right) \\
& =\left(\alpha_{2}^{2}+v \lambda_{m}^{2}\right)\left[\alpha_{1}^{2}-(2-v) \lambda_{m}^{2}\right] \alpha_{1} \tan \left(\alpha_{2} L_{e}\right) .
\end{aligned}
$$

(6) relates the residual stress to the buckling wavelength and the etching length. For a given etching length and buckling wavelength, the residual stress is determined by (6). Thus, the buckling measurement provides a method to evaluate the residual stress within a small region of the order of the microbeam length. On the other hand, if we know the residual stress, (6) can be used to estimate the etching length or the buckling wavelength. In the present study, the etching length was measured to be $10 \mu \mathrm{m}$ for the first mode buckling. Using this datum, the residual stress was then evaluated from (6) to be $300 \mathrm{MPa}$ in compression. Comparing this value of $300 \mathrm{MPa}$ with $315 \mathrm{MPa}$ obtained from the curvature measurement, it is clear that the buckling measurement yields a slightly smaller value and we believe that the value from the buckling measurement is more reliable because the curvature measurement provides a mean value averaged over the entire wafer.

(3) describes the three-dimensional profile of the first mode of buckling. Accordingly, the threedimensional profile of the second mode of buckling can be approximately described by:

$$
\begin{aligned}
w= & {\left[\sinh \left(\alpha_{1} y\right)+\frac{\left(\alpha_{1}^{2}-v \lambda_{m}^{2}\right) \sinh \left(\alpha_{1} L_{e}\right)}{\left(\alpha_{2}^{2}+v \lambda_{m}^{2}\right) \sin \left(\alpha_{2} L_{e}\right)} \sin \left(\alpha_{2} y\right)\right] } \\
& \times\left[A_{I}\left(1+\sin \left(\lambda_{m} x\right)\right)\right. \\
& \left.-\frac{h_{s}}{\sinh \left(\alpha_{1} L_{e}\right)+\frac{\left(\alpha_{1}^{2}-\nu \lambda_{m}^{2}\right) \sinh \left(\alpha_{1} L_{e}\right)}{\alpha_{2}^{2}+\nu \lambda_{m}^{2}}}\right] .
\end{aligned}
$$

Using the buckling data for the two beams illustrated in figures 4 and 5, we plot in figures 6(a)-6(d) the calculated, three-dimensional profiles and plan views for the first and second modes of buckling. Comparing figure 6(b) to figure 3(a) and figure 6(d) to figure 3(b), we may conclude that the buckling profiles are approximately described by (3) and (7) for the first and second modes of buckling.

If the clamped-edge boundary conditions along the etch front $y=0$ are adopted the displacement along the $z$ axis, $w$, for the first mode buckling is given by

$$
\begin{aligned}
w & =A_{I I}\left[\sinh \left(\alpha_{1} y\right)-\frac{\alpha_{1}}{\alpha_{2}} \sin \left(\alpha_{2} y\right)\right. \\
& \left.+C\left(\cosh \left(\alpha_{1} y\right)-\cos \left(\alpha_{2} y\right)\right)\right] \sin \left(\lambda_{m} x\right)
\end{aligned}
$$

In this case, the amplitude of buckling has the form of

$$
\begin{aligned}
A & =A_{I I}\left[\sinh \left(\alpha_{1} L_{e}\right)-\frac{\alpha_{1}}{\alpha_{2}} \sin \left(\alpha_{2} L_{e}\right)\right. \\
& \left.+C\left(\cosh \left(\alpha_{1} L_{e}\right)-\cos \left(\alpha_{2} L_{e}\right)\right)\right]
\end{aligned}
$$

and (8) is subject to the condition of

$$
\begin{aligned}
& {\left[\left(\alpha_{1}^{3} \sinh \left(\alpha_{1} L_{e}\right)-\alpha_{2}^{3} \sin \left(\alpha_{2} L_{e}\right)\right)\right.} \\
& \left.\quad-(2-v) \lambda_{m}^{2}\left(\alpha_{1} \sinh \left(\alpha_{1} L_{e}\right)+\alpha_{2} \sin \left(\alpha_{2} L_{e}\right)\right)\right] \\
& \quad \times\left[\left(\alpha_{1}^{2} \sinh \left(\alpha_{1} L_{e}\right)+\alpha_{1} \alpha_{2} \sin \left(\alpha_{2} L_{e}\right)\right)\right. \\
& \left.\quad-v \lambda_{m}^{2}\left(\sinh \left(\alpha_{1} L_{e}\right)-\frac{\alpha_{1}}{\alpha_{2}} \sin \left(\alpha_{2} L_{e}\right)\right)\right] \\
& \quad-\left[\left(\alpha_{1}^{3} \cosh \left(\alpha_{1} L_{e}\right)+\alpha_{1} \alpha_{2}^{2} \cos \left(\alpha_{2} L_{e}\right)\right)\right. \\
& \left.\quad-(2-v) \lambda_{m}^{2}\left(\alpha_{1} \cosh \left(\alpha_{1} L_{e}\right)-\alpha_{1} \cos \left(\alpha_{2} L_{e}\right)\right)\right] \\
& \quad \times\left[\left(\alpha_{1}^{2} \cosh \left(\alpha_{1} L_{e}\right)+\alpha_{2}^{2} \cos \left(\alpha_{2} L_{e}\right)\right)\right. \\
& \left.-v \lambda_{m}^{2}\left(\cosh \left(\alpha_{1} L_{e}\right)-\cos \left(\alpha_{2} L_{e}\right)\right)\right]=0 .
\end{aligned}
$$

Using the same experimental data, the residual stress evaluated from (11) is $787 \mathrm{MPa}$ in compression, about $470 \mathrm{MPa}$ higher than $315 \mathrm{MPa}$ obtained from the curvature measurements. Based on (8) we calculate and plot the three-dimensional profiles and plan views for the first mode buckling, as shown in figures 7(a) and 7(b). It seems difficult to distinguish figures 7(a) and 7(b) correspondingly from figures 6(a) and 6(b). We see the difference, however, if we take $\sin \left(\lambda_{m} x\right)=1$ and plot the displacement, $w$, as a function of $y$. Figure 8 shows these three curves: curve SS is calculated from (3), curve CL is calculated from (8) and curve $\mathrm{C}$ is measured along tracing path $\mathrm{C}$, i.e., curve $\mathrm{C}$ in figure 4(b). Since the clamped-edge boundary conditions do not allow any rotation at the etch front $y=0$, curve SS drops much faster than curve CL when $y$ approaches zero. It is interesting to note that curve $\mathrm{C}$ is located between curves SS and CL. This means that the real boundary conditions along the etch front cannot be exactly described by either the simply supported or the clamped boundary conditions.

\section{Concluding remarks}

Three modes of buckling patterns, mode I, the sinusoidal half waves, mode II, the constrained sinusoidal half waves, and mode III, the classical mode, were observed to evolve during the sacrificial etch of polysilicon beams. The buckling patterns, which are the result of the compressive residual stress, developed when the microbeams were still immersed within the etching solution. Theoretical calculations based on either simply supported or clamped boundary conditions were performed and compared with

where

$$
C=-\frac{\left[\alpha_{1}^{3} \cosh \left(\alpha_{1} L_{e}\right)+\alpha_{1} \alpha_{2}^{2} \cos \left(\alpha_{2} L_{e}\right)\right]-(2-v) \lambda_{m}^{2}\left[\alpha_{1} \cosh \left(\alpha_{1} L_{e}\right)-\alpha_{1} \cos \left(\alpha_{2} L_{e}\right)\right]}{\left[\alpha_{1}^{3} \sinh \left(\alpha_{1} L_{e}\right)-\alpha_{2}^{3} \sin \left(\alpha_{2} L_{e}\right)\right]-(2-v) \lambda_{m}^{2}\left[\alpha_{1} \sinh \left(\alpha_{1} L_{e}\right)+\alpha_{2} \sin \left(\alpha_{2} L_{e}\right)\right]} .
$$




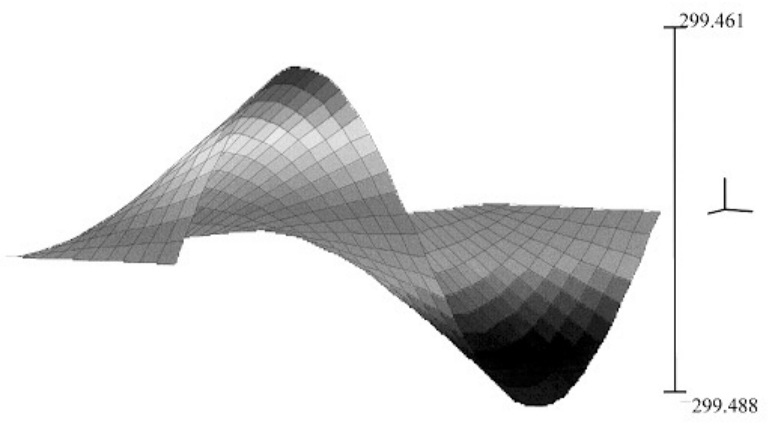

(a)

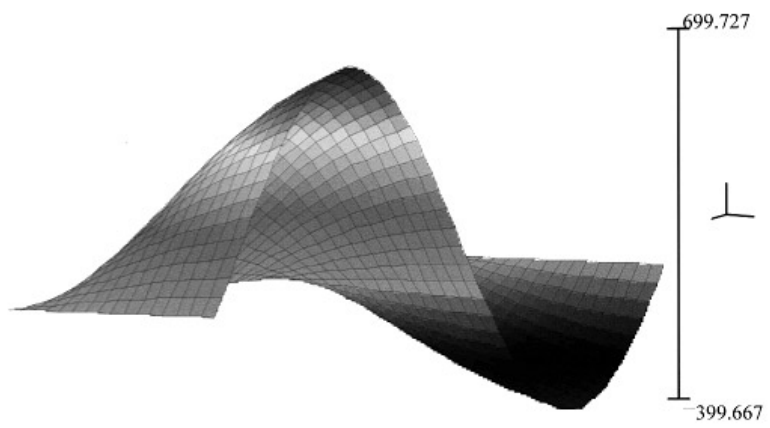

(c)

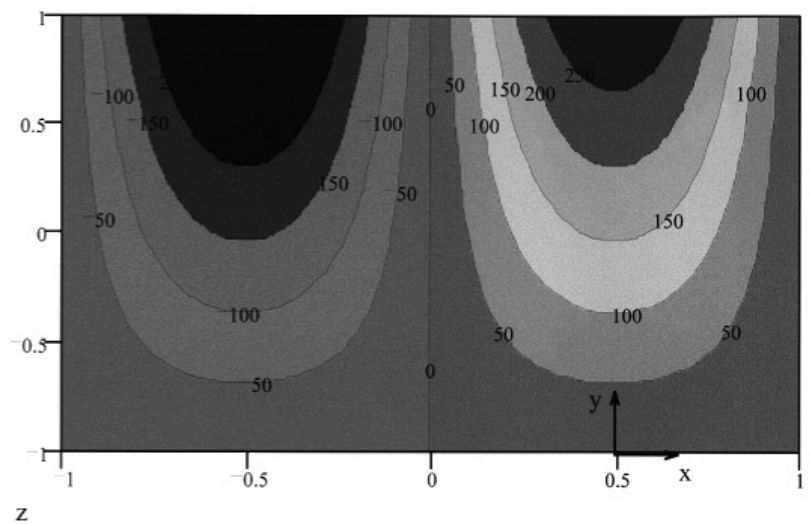

(b)

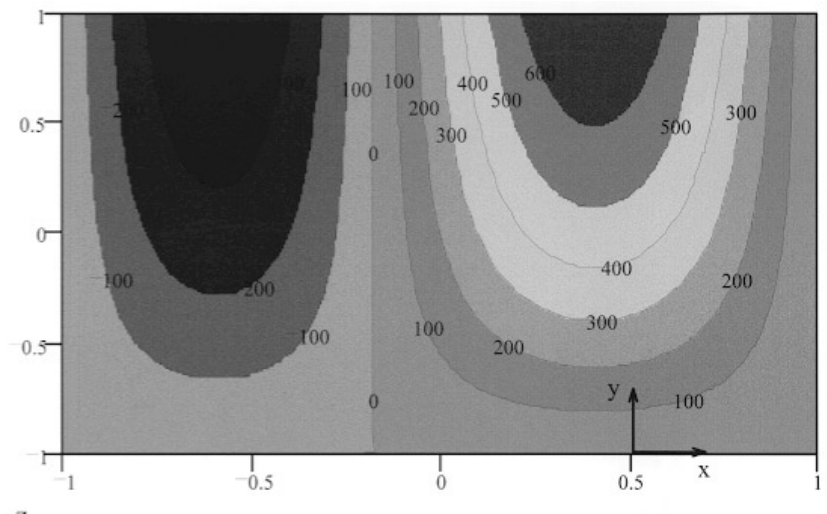

(d)

Figure 6. (a) Three-dimensional profile of the first mode buckling described by (3) (in nm). (b) Plan view of the first mode buckling described by (3) (in $\mathrm{nm}$ ). (c) Three-dimensional profile of the second mode buckling described by (7) (in $\mathrm{nm}$ ). (d) Plan view of the second mode buckling described by (7) (in $\mathrm{nm}$ ).

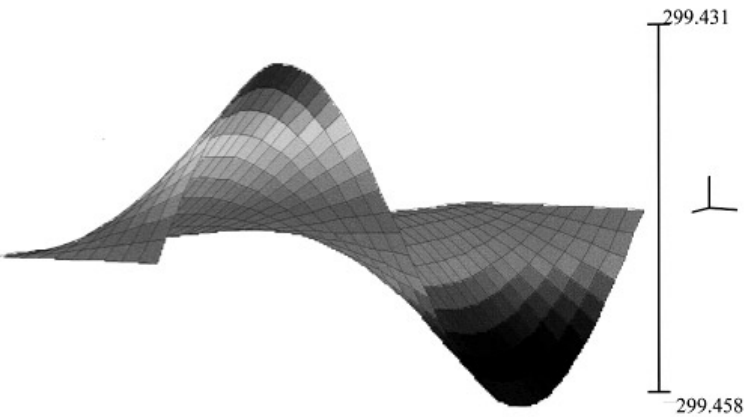

(a)

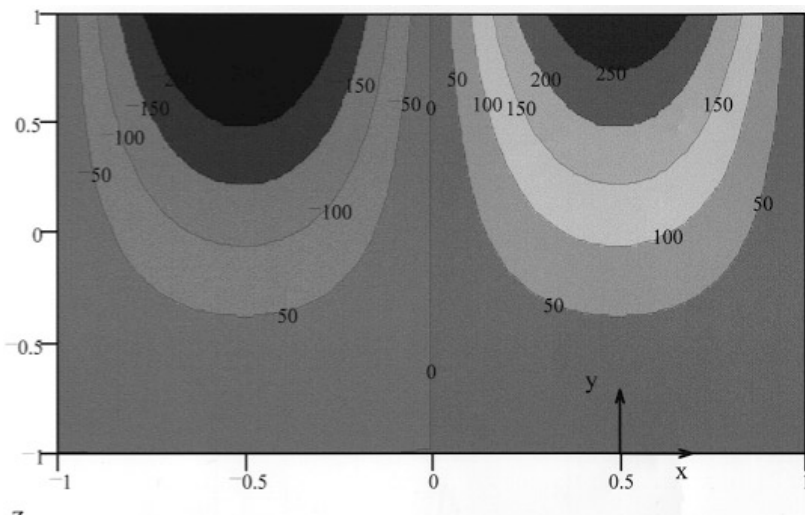

(b)

Figure 7. (a) Three-dimensional profile of the first mode buckling described by (8) (in nm). (b) Plan view of the first mode buckling described by (8) (in $\mathrm{nm}$ ).

the experimental measurements. The real profile of mode I buckling is located at the middle of the calculated profiles based on the simply supported and clamped boundary conditions, indicating that the real boundary conditions are mixed ones of the simply supported and clamped. Furthermore, the calculated residual stress based on the simply supported boundary conditions is $300 \mathrm{MPa}$ in compression, close to the value of $315 \mathrm{MPa}$ measured by the curvature method, while it is $787 \mathrm{MPa}$ using the clamped boundary conditions. This means that the weight of the simply supported boundary conditions should be higher than that of the clamped in the mixed boundary conditions for the polysilicon microbeams. For the mode II buckling, the approximate formula, i.e., (7), that describes the profile of the mode II buckling, provides a clue to the derivation of an exact solution. 


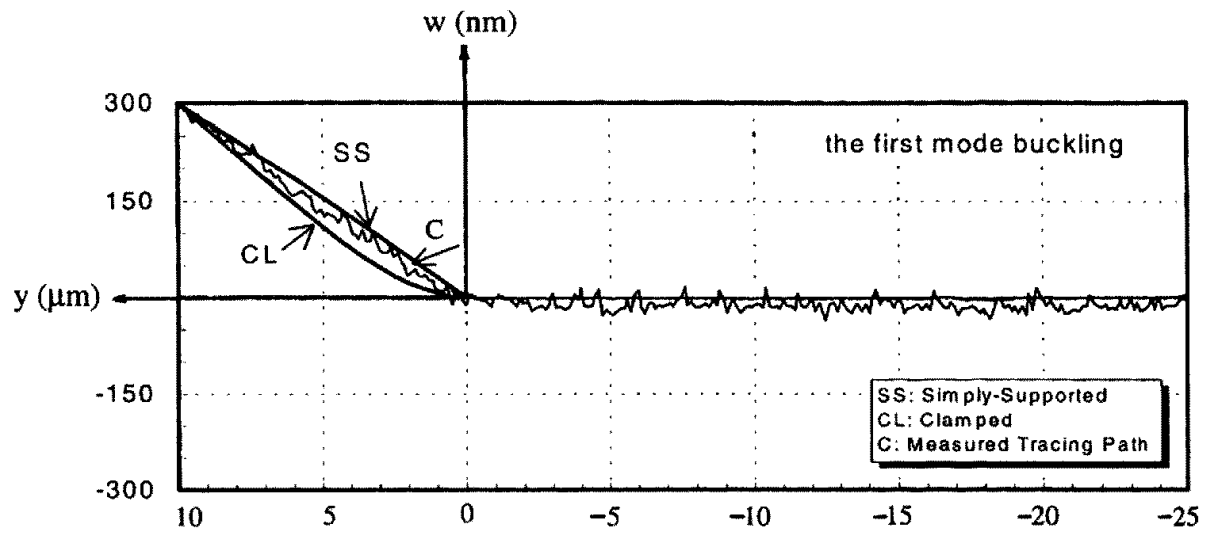

Figure 8. Deflection displacement, $w$, as a function of the distance from the free edge: curve SS calculated from (3), curve CL calculated from (8) and curve $C$ measured along tracing path $C$ in figure 4(a).

\section{Acknowledgments}

This work was sponsored by the Hong Kong Research Grants Council through RGC grant HKUST713/95E. The authors are also grateful to Professor Pin Tong for useful discussion.

\section{References}

[1] Fan L S, Tai Y-C and Muller R S 1988 Integrated movable micromechanical structures for sensors and actuators IEEE Trans. Electron Devices ED-35 724-30

[2] Hutchinson J W, Thouless M D and Liniger E G 1992
Growth and configurational stability of circular, buckling-driven film delaminations Acta Metall. Mater. 40 295-308

[3] Jensen H M and Thouless M D 1995 Buckling instability of straight edge cracks ASME Trans. J. Appl. Mech. 62 $620-5$

[4] Vlassak J J and Nix W D 1992 A new bulge test technique for the determination of Young's modulus and Poisson's ratio of thin films J. Mater. Res. 7 3242-9

[5] Timoshenko S P and Gere J M 1961 Theory of Elastic Stability 2nd edn (Singapore: McGraw-Hill)

[6] Zhang X, Zohar Y and Zhang T-Y Blister-like local buckling of polysilicon microbeams Int. Mechanical Engineering Congress Exposition (Anaheim, CA, 1998) 